(C) Dereito Vol.27, no:43-60 (Xullo-Decembro, 2018) • ISSN 1132-9947

\title{
LA NUEVA LEY DE CONTRATOS DEL SECTOR PÚBLICO: ¿UN INSTRUMENTO PARA LOGRAR LA IGUALDAD EFECTIVA?
}

The new public sector contracts law: an instrument to achieve effective equality?

DOI: http://dx.doi.org/10.15304/dereito.27.2.5344

\section{SuSANA SOUTO MARTÍN}

Doctoranda en Creatividade e Innovación Social e Sostible

Universidade de Vigo

ssouto@uvigo.es

\section{Resumen}

La igualdad ha tomado, en los últimos años, gran relevancia en el plano internacional, nacional y supranacional, tal y como muestra el desarrollo de políticas de igualdad de género de las últimas décadas.

La contratación pública no es un fin en sí misma, sino una herramienta que las Administraciones públicas pueden utilizar para desarrollar políticas públicas, entre las que destacan las de tipo social

Este artículo trata de aportar una visión sobre la contratación pública como actor clave en la ejecución de las políticas de igualdad.

Palabras clave: Contratación pública, contratos públicos, cláusulas sociales, igualdad de género.

\section{Abstract}

In recent years the equality has taken great relevance at the international, national and supranational level, as shown in the development of gender equality policies during the latest decades.

Public contracts are not a purpose by themself, but a tool that Public Administrations can use to develope public policies, including social ones. This article seeks a vision about the public procurement as a key player in the development, planning and implementation of equality policies.

Keywords: Public procurement, public contracts, social clauses, equal opportunities.

\section{SUMARIO}

1.INTRODUCTION.; -2. GENDER EQUALITY.; -3.PUBLIC PROCUREMENT.; -4.COMPARATIVE TRLCSP VS LCSP.; -4.1 .SOCIAL CONTENT CAUSES OF PROHIBITION TO CONTRACT.; -4.2.CONDITIONS OF APTITUDE TO CONTRACT.; -4.3.THE ISSUE OF THE CONTRACT.; -4.4.TENDER EVALUATION CRITERIA.; -4.5.SOCIAL CLAUSES AS ADJUDICATION CRITERIA.; -4.6.SOCIAL CLAUSES AS TIE-BREAKER CRITERIA.; 4.7.SPECIAL IMPLEMENT CONDITIONS.; -4.8.EXCEPTIONS.; 5.LIMITATIONS OF THIS STUDY.; -6.CONCLUSIONS.; -7.BIBLIOGRAPHY.

Recibido: 04/09/2018. Aceptado: 05/12/2018. 


\section{SUMMARY}

1.INTRODUCTION.; -2.GENDER EQUALITY.; -3.PUBLIC PROCUREMENT. ; 4.COMPARATIVE TRLCSP VS LCSP.; -4.1. SOCIAL CONTENT CAUSES OF PROHIBITION TO CONTRACT.; -4.2.CONDITIONS OF APTITUDE TO CONTRACT.; -4.3.THE ISSUE OF THE CONTRACT.; -4.4. TENDER EVALUATION CRITERIA.; - 4.5.SOCIAL CLAUSES AS ADJUDICATION CRITERIA.; -4.6. SOCIAL CLAUSES AS TIE-BREAKER CRITERIA.; -4.7. SPECIAL IMPLEMENT CONDITIONS.; -4.8.EXCEPTIONS.; -5. LIMITATIONS OF THIS STUDY.; -6.CONCLUSIONS. ; -7.BIBLIOGRAPHY.

\section{INTRODUCCIÓN}

La Constitución española, en su art. 103.1, establece el principio esencial de funcionamiento de las Administraciones Públicas determinando que estas han de servir con objetividad a los intereses generales. Se refiere así al interés general como concepto jurídico indeterminado que, en virtud de las potestades que las Administraciones Públicas tienen atribuidas, debe trasladarse a los intereses públicos concretos $^{1}$. De la materialización de este mandato se encarga la gestión pública.

A lo largo de la historia hemos vivido, o tal vez sufrido, diversos modelos de gestión pública hasta llegar al nuevo paradigma de nuestro tiempo: la gobernanza: pero ¿qué es la gobernanza? ${ }^{2}$ J.Ma. VALLĖS I CASADEVALL la define como "la coordinación social que nace de la interacción constante entre agentes de todo tipo ${ }^{\prime \prime 3}$ y L. F. AGUILAR VILLANUEVA explicita que en ese sentido "la dirección social eficaz y valiosa implica un pluricentrismo directivo, una dirigencia colectiva interdependiente y asociada" ${ }^{\prime 4}$. Esto es, la conjunción de una multitud de agentes para conseguir un objetivo final, que no es otro que lograr que la gestión de lo público no sea algo ajeno a sus usuarios.

Atendiendo a este nuevo modelo de gestión la contratación pública, como una de las actividades principales de las Administraciones Públicas, puede y debe ser una técnica que permita conseguir objetivos sociales, ambientales o de investigación, en la convicción de que los mismos

\footnotetext{
${ }^{1}$ M. BURZACO SAMPER. "Contrataciones públicas socialmente responsables: la necesidad de reconsiderar el potencial de la contratación pública en la consecución de objetivos sociales", CIRIEC España, Revista de Economía Pública, Social y Cooperativa, 86, 2016, pp. 281-310.

2 Para una aproximación al concepto: J. SUBIRATS HUMET. "Si la respuesta es gobernanza, ¿cuál es la pregunta?: factores de cambio en la política y en las políticas", Ekonorniaz. Revista vasca de economía, 24, 2010, pp. 246-269; E.J.VARELA ÁLVAREZ. coord. Dirección pública profesional: lecturas para xestionar a era da gobernanza. Escola Galega de Administración Pública. Santiago de Compostela, 2009; F. MORATA TIERRA. "Regiones y Gobernanza Multinivel en la Unión Europea", en F. MORATA TIERRA (Ed.). Gobernanza Multinivel en la Unión Europea, Tirant lo Blanch, Valencia, 2004, pp. 19-50.

3 J.Ma. VALLĖS I CASADEVALL. Ciencia Política. Una Introducción, Ariel, Barcelona, 2006. p.429

${ }^{4}$ L. F. AGUILAR VILLANUEVA. GOBERNANZA: El nuevo proceso de gobernar, Fundación Friedrich Naumanm para la libertad, Ciudad de México, 2010, p.37
} 
comportan una adecuada comprensión de cómo deben canalizarse los fondos públicos. ${ }^{5}$

Los contratos públicos, como política pública que "de facto" son, deben contribuir a construir una realidad más humana y una mejor sociedad para la población ${ }^{6}$. Como señalaba M.C. CONTE, especialista de la Comisión Interamericana de Mujeres (CIM): "la contratación pública puede ser una herramienta de política muy útil para contribuir con el empoderamiento económico de las mujeres, con una transformación socio-económica, e inclusive para avanzar los objetivos más amplios de igualdad. ${ }^{\prime 7}$

El nivel de desigualdad de género no es comparable con el que existía hace 50 años. No obstante, continuamos muy lejos del objetivo de una sociedad plenamente igualitaria. En momentos de bonanza económica la brecha es importante, pero es en los de crisis en los que esta se vuelve sangrante. Basten como ejemplo las cifras de paro en España durante el tercer trimestre de 2017 . La tasa fue del $16.38 \%$, pero mientras el paro masculino fue del $14.80 \%$, el femenino supuso un $18.21 \%{ }^{8}$ Parece evidente la necesidad de políticas de empleo que corrijan esta situación de desigualdad.

La contratación pública socialmente responsable es una herramienta decisiva en las políticas de empleo que puede y debe contribuir a la inserción laboral de colectivos excluidos y que, como señala J. BERNETE GARCÍA $^{9}$ conecta perfectamente con uno de los ejes de la Estrategia Europea de «Inclusión Activa» que hace referencia a la vinculación con medidas activas del mercado laboral propiciando mercados laborales inclusivos ${ }^{10}$.

5 M. Á. BERNAL BLAY. "Hacia una contratación pública socialmente responsable: Las oportunidades de la ley 30/2007, de 30 de octubre de contratos del sector público". Revista Aragonesa De Administración Pública, 10, 2008, pp. 211-252.

6 J. MIRANZO DÍAZ. "Hacia una administración pública sostenible: novedades en la legislación europea de contratación pública" Actualidad Jurídica Ambiental, 64, 2017, pp. $1-40$

7 M.C. CONTE. "Potencial impacto de las compras públicas para el empoderamiento de las mujeres" en "Promoviendo el Desarrollo Inclusivo: Incrementando la Participación de la Mujer en Contratación Pública" Banco Interamericano de Desarrollo - Washington, D.C. 26-27 de mayo de 2015, p.3

${ }^{8}$ Fuente INE http://www.ine.es/infografias/tasasepa/desktop/tasas.html?t=0\&lang=es fecha de consulta $18 / 2 / 18$

9 J. BERNETE GARCÍA. "Cláusulas sociales en la contratación pública: Nuevos instrumentos para el fomento del empleo a nivel local". Cuadernos De Trabajo Social, 26, 2013, pp.85-94.

10 Véase, en este sentido, el estudio sobre la evolución de la inclusión de clausulas sociales en el derecho de la Unión Europea de J.M. MIRANDA BOTO. "Contratación pública y cláusulas de empleo y condiciones de trabajo en el derecho de la Unión Europea" Lex Social, vol. 6, 2, 2016, pp. 69-91; y, sin ánimo de exhaustividad: S. RODRÍGUEZ ESCANCIANO. Cláusulas sociales y licitación pública: análisis jurídico. Instituto Andaluz de Administración Pública, Sevilla, 2009; J. MORCILLO MORENO. "Las clausulas sociales en la contratación pública como garantía frente al dumping social intracomunitario". Revista Aragonesa De Administración Pública, 47-48, 2016, p.p. 180209; M. SOLÓRZANO GARCÍA, J. NAVÍO MARCO y R. CONTRERAS COMECHE. "Incorporación de valor social a la contratación pública en España: Situación y 
El objetivo de este trabajo será analizar si la nueva Ley de Contratos del Sector Público ${ }^{11}$ (en adelante LCSP) ha supuesto un avance con respecto a su predecesora, Texto Refundido de la Ley de Contratos de Sector Público ${ }^{12}$ (en adelante TRLCSP) en lo que se refiere a la utilización de la contratación pública como instrumento corrector de la desigualdad de género.

El método de investigación será el estudio comparado. Para ello, teniendo muy en cuenta la Ley Orgánica para la igualdad efectiva de hombres y mujeres ${ }^{13}$ (en adelante LOI) analizaremos las novedades introducidas en la LCSP comparándolas con lo que establecía el TRLCSP en lo que a Contratación Pública Responsable se refiere, centrándonos en las cláusulas sociales $y$, muy especialmente, en las referidas a igualdad de género.

\section{IGUALDAD DE GÉNERO}

Entendemos por Discriminación positiva, aquellas medidas que se dirigen a un grupo determinado encaminadas a suprimir una discriminación, aunque ello suponga una ventaja respecto a otro grupo social. Este concepto forma parte de otro más amplio, el de Acción positiva, que se encamina a remover obstáculos a un grupo desfavorecido sin perjudicar a otro ${ }^{14}$.

La LOI constituye un intento de que estas acciones positivas contribuyan a la consecución de la igualdad efectiva de hombres y mujeres en lo que al ámbito de la vida pública de las personas se refiere. Para ello señala aquellos escenarios en los que estas son necesarias ${ }^{15}$. Las referidas a la contratación del Sector Público se recogen en dos artículos de la LOI: arts. 33 y 34.

Art. 33 "Las Administraciones públicas, en el ámbito de sus respectivas competencias, a través de sus órganos de contratación y, en relación con la ejecución de los contratos que celebren, podrán establecer condiciones especiales con el fin de promover la igualdad entre mujeres y hombres en el mercado de trabajo, de acuerdo con lo establecido en la legislación de contratos del sector público."

perspectivas". Revista De Estudios De La Administración Local Y Autonómica, 4,2015, pp. 41-50

11 Ley 9/2017, de 8 de noviembre, de Contratos del Sector Público, por la que se transponen al ordenamiento jurídico español las Directivas del Parlamento Europeo y del Consejo 2014/23/UE y 2014/24/UE, de 26 de febrero de 2014.BOE 272, de 9 de noviembre de 2017

12 Real Decreto Legislativo 3/2011, de 14 de noviembre, por el que se aprueba el texto refundido de la Ley de Contratos del Sector Público

${ }^{13}$ Ley Orgánica $3 / 2007$, de 22 de marzo, para la igualdad efectiva de mujeres y hombres BOE 71 de 23/3/2007

14 S. LESMES ZABALEGUI. "Contratación pública y discriminación positiva. Cláusulas sociales para promover la igualdad de oportunidades entre mujeres y hombres en el mercado laboral". Lan harremanak: Revista de relaciones laborales, 13,2015, pp.53-86.

15 S. MONTENEGRO LEZA. "El antes y el después de la Ley Orgánica 3/ 2007", REDUR,14, 2016, pp. 117-136. 
Resulta relevante en la redacción de este artículo la remisión a la legislación de contratos del sector público, lo que deja el mismo en una mera declaración de intenciones. No se trata de un imperativo, sino de dejar abierta la posibilidad. Algo más tajante es la redacción del artículo siguiente; a saber

Art 34

1. "Anualmente, el Consejo de Ministros, a la vista de la evolución e impacto de las políticas de igualdad en el mercado laboral, determinará los contratos de la Administración General del Estado y de sus organismos públicos que obligatoriamente deberán incluir entre sus condiciones de ejecución medidas tendentes a promover la igualdad efectiva entre mujeres y hombres en el mercado de trabajo, conforme a lo previsto en la legislación de contratos del sector público.

En el Acuerdo a que se refiere el párrafo anterior podrán establecerse, en su caso, las características de las condiciones que deban incluirse en los pliegos atendiendo a la naturaleza de los contratos y al sector de actividad donde se generen las prestaciones"

2. Los órganos de contratación podrán establecer en los pliegos de cláusulas administrativas particulares la preferencia en la adjudicación de los contratos de las proposiciones presentadas por aquellas empresas que, en el momento de acreditar su solvencia técnica o profesional, cumplan con las directrices del apartado anterior, siempre que estas proposiciones igualen en sus términos a las más ventajosas desde el punto de vista de los criterios objetivos que sirvan de base a la adjudicación y respetando, en todo caso, la prelación establecida en el apartado primero de la disposición adicional octava del Texto Refundido de la Ley de Contratos de las Administraciones Públicas, aprobado por Real Decreto Legislativo 2/2000, de 16 de junio."

Vemos, no obstante, que la remisión a la legislación de contratos del sector público se mantiene. Será, por tanto, la nueva LCSP la que podrá o no dotar de contenido estos dos artículos.

Cabe señalar que _durante la vigencia del TRLCSP_ diversas sentencias se han basado en estos artículos; sirva como ejemplo la dictada por el Tribunal Supremo (TS) en julio de 2012 en relación a un contrato de obras de una estación de aguas residuales que, entre los criterios de adjudicación y en aplicación de dichos artículos, había otorgado una puntuación por el porcentaje de personal femenino fijo en la empresa. El TS sentencia que este es un elemento para otorgar una preferencia en la adjudicación solamente en el caso de que, con la aplicación de los criterios objetivos que deben regir en la adjudicación, se produzca una situación de igualdad o empate entre dos o más ofertas o proposiciones. ${ }^{16}$

\footnotetext{
${ }^{16}$ Tribunal Supremo (Sala de lo Contencioso Administrativo, Sección 7) Sentencia de 17 de julio de 2012
} 


\section{CONTRATACIÓN PÚBLICA}

Mientras las primeras generaciones de directivas identificaban el precio más bajo como el único parámetro de evaluación de las ofertas, y tenían como único objetivo, al regular la contratación pública, la adquisición de bienes y servicios; esta última generación, sin olvidar los objetivos que para la contratación pública aparecen en el Tratado de Funcionamiento de la Unión Europea (TFUE) ${ }^{17}$, refleja que esta no puede ya ser considerada como un fin en sí misma, sino como una herramienta para lograr una aplicación efectiva de las políticas públicas básicas. ${ }^{18}$

La Directiva 2014/24/UE señala como objetivo, en su Considerando 2, "permitir que los contratantes utilicen mejor la contratación pública en apoyo de objetivos sociales comunes". Además, en el art. 18.2, que debe ser observado durante todo el procedimiento y la vida del contrato, impone la obligación a los Estados Miembros de

"tomar las medidas pertinentes para garantizar que, en la ejecución de contratos públicos, los operadores económicos cumplen las obligaciones aplicables en materia medioambiental, social o laboral establecidas en el Derecho de la Unión, el Derecho nacional, los convenios colectivos o por las disposiciones de Derecho internacional medioambiental, social y laboral enumeradas en el anexo $X^{\prime \prime}$.

De las disposiciones enumeradas en este anexo, tienen relevancia para este estudio las referidas a los Convenios de la Organización Internacional del Trabajo (OIT) $100^{19}$ y $111^{20}$. El primero, de 1951 , relativo a la igualdad de remuneración entre la mano de obra masculina y la mano de obra femenina por un trabajo de igual valor y, el segundo, de 1958, sobre la discriminación en materia de empleo y ocupación.

Parece, por tanto, que en este sentido, no se refleja en esta Directiva nada más allá de la obligación de los Estados Miembros de cumplir con la legislación vigente. Sin embargo la referencia a los objetivos sociales comunes nos remite a un concepto que si podemos considerar novedoso; la contratación pública socialmente responsable (en adelante CPSR)

En el año 2001 la propia Comisión Europea, en una Comunicación interpretativa señalaba

"las actuales Directivas sobre contratos públicos no contemplan expresamente la posibilidad de que se persigan objetivos de política social con motivo del procedimiento de adjudicación de un contrato público", no obstante, "la actual normativa comunitaria de contratación pública ofrece todo un abanico de posibilidades que, convenientemente aprovechadas, permiten alcanzar el resultado buscado." 21

\footnotetext{
17 Tratado de Funcionamiento de la Unión Europea Diario Oficial $n^{\circ}$ C 326 de 26/10/2012 p. $0001-0390$

18 J. MIRANZO DÍAZ. "Hacia una administración... op.cit.

${ }^{19}$ OIT C100 - Convenio sobre igualdad de remuneración, 1951 (núm. 100)

${ }^{20}$ OIT C111 - Convenio sobre la discriminación (empleo y ocupación), 1958 (núm. 111)

21 COMISIÓN EUROPEA. Comunicación interpretativa de la Comisión sobre legislación comunitaria de contratos públicos y las posibilidades de integrar aspectos sociales en dichos contratos Bruselas, 15.10.2001.COM (2001) 566 final
} 
La preocupación, a todas luces, ya estaba ahí. En el año 2010, la Comisión Europea define ampliamente el concepto de contrataciones públicas socialmente responsables:

"Las «CPSR» se refieren a las operaciones de contratación que tienen en cuenta uno o más de los siguientes aspectos sociales: oportunidades de empleo, trabajo digno, cumplimiento con los derechos sociales y laborales, inclusión social (incluidas las personas con discapacidad), igualdad de oportunidades, diseño de accesibilidad para todos, consideración de los criterios de sostenibilidad, incluidas las cuestiones de comercio ético y un cumplimiento voluntario más amplio de la responsabilidad social de las empresas (RSE), a la vez que se respetan los principios consagrados en el Tratado de la Unión Europea (TUE) y las Directivas de contratación 122 .

Así la Directiva 2014/24/UE viene a dar respuesta a una preocupación cuya importancia señala la propia Comisión cuando, en su Comunicación de 2 de diciembre de $2015^{23}$, señala "La contratación pública representa una gran proporción del consumo europeo (casi el $20 \%$ del PIB de la UE)" reconociendo, por tanto, el peso que esta tiene en cualquier política pública.

De la imposición de transposición esta, y del resto de la cuarta generación de Directivas de contratación de febrero de $2014^{24}$ nace _tal y como se recoge en su título_ la LCSP. ${ }^{25}$

22 COMISIÓN EUROPEA. Adquisiciones sociales. Una guía para considerar aspectos sociales en las contrataciones públicas. Luxemburgo: Dirección General de Empleo, Asuntos Sociales e Igualdad de Oportunidades. $\operatorname{COM}(2010)$ \{sec (2010) 1258 final\} p. 7

${ }^{23}$ COMISIÓN EUROPEA. Comunicación de la Comisión al Parlamento Europeo, al Consejo, al Comité económico y social europeo y al Comité de las regiones "Cerrar el círculo: un plan de acción de la UE para la economía circular" COM (2015) 614 final, de 2 de diciembre de 2015

${ }^{24}$ Directivas, publicadas en el Diario Oficial de la Unión Europea de 28 de marzo de 2014:

- Directiva 2014/23/UE del Parlamento Europeo y del Consejo de 26 de febrero de 2014 relativa a la adjudicación de contratos de concesión. DOUE núm. L 94/1.

- Directiva 2014/24/UE del Parlamento Europeo y del Consejo de 26 de febrero de 2014 sobre contratación pública y por la que se deroga la Directiva 2004/18/CE. DOUE núm. L $94 / 65$.

- Directiva 2014/25/UE del Parlamento Europeo y del Consejo de 26 de febrero de 2014 relativa a la contratación por entidades que operan en los sectores del agua, la energía, los transportes y los servicios postales y por la que se deroga la Directiva 2004/17/CE. DOUE núm. L 94/243.

${ }^{25}$ Vid J. M. GIMENO FELIU. "La reforma comunitaria en materia de contratos públicos y su incidencia en la legislación española. Una visión desde la perspectiva de la integridad", en libro colectivo Las Directivas de Contratación Pública, número monográfico especial Observatorio de los Contratos Públicos 2014, Aranzadi, Cizur Menor. pp. 37-105.; M.M. RAZQUIN LIZARRAGA. "Las nuevas Directivas sobre contratación pública de 2014: aspectos clave y propuestas para su transformación en España", RAP,196, 2015, pp. 9713 


\section{COMPARATIVA TRLCSP VS LCSP}

Ya en el artículo 1.3 LCSP se establece que:

"en toda contratación pública se incorporarán de manera transversal y preceptiva criterios sociales y medioambientales siempre que guarde relación con el objeto del contrato, en la convicción de que su inclusión proporciona una mejor relación calidad-precio en la prestación contractual, así como una mayor y mejor eficiencia en la utilización de los fondos públicos .Igualmente se facilitará el acceso a la contratación pública de las pequeñas y medianas empresas, así como de las empresas de economía social"

Este precepto, que no formaba parte del proyecto de Ley, fue aprobado por unanimidad por la Ponencia constituida en el Congreso de los Diputados ${ }^{26}$. Lo que se persigue con su inclusión es conformar los criterios sociales y medioambientales como un principio rector de la contratación pública. Se pasa de este modo, de una visión presupuestaria de la contratación pública, a una perspectiva instrumental de implementación de políticas públicas ${ }^{27}$.

El art. 28.2 LCSP, que viene a substituir al art. 22 del TRLCSP añade a este que las entidades del sector público valorarán la incorporación de consideraciones sociales, medioambientales y de innovación como aspectos positivos en los procedimientos de contratación pública.

Desde aquí, pasaremos a analizar la concreción de este principio rector a lo largo del articulado de la Ley distinguiendo su repercusión en las dos fases más significativas de procedimiento de contratación: selección y adjudicación.

\subsection{Causas de prohibición de contratar de contenido social}

Las causas de prohibición para contratar se recogen en el art. 71 LCSP. En lo que a contenido social se refiere, este no introduce ninguna novedad con respecto a su precedente, el art. 60 TRLCSP, a excepción del apartado 1.d dónde se recoge el supuesto de incumplimiento del requisito mínimo de trabajadores con discapacidad.

Este sería, en buena lógica, el lugar en que si verdaderamente se quisiese hacer una política pública efectiva debería haberse incluido la prohibición expresa de contratar con el sector público de aquellas empresas que no cumpliesen con la LOI.

\subsection{Condiciones de aptitud para contratar}

La LCSP mantiene, en genera, lo estipulado en el TRLCSP aportando algunas novedades. Por ejemplo, introduce entre las condiciones de aptitud para contratar el de la "solvencia social" del licitador desde el

26 I. GALLEGO CÓRCOLES. "La integración de cláusulas sociales, ambientales y de innovación en la contratación pública". Documentación Administrativa: Nueva Época, 4, 2017, pp. 90-113.

27 J. M.GIMENO FELIU "La transposición de las directivas de contratación pública en España: Una primera valoración de sus principales novedades". Documentación Administrativa. Nueva Época, 4, 2017, pp.7-30. 
punto de vista técnico o profesional, y la posibilidad de excluir de la participación en contratos públicos a empresas con valores sociales anormalmente bajos. En este sentido el art. 90.3 LCSP, referido a los contratos de servicios, estipula que si el objeto contractual requiriese aptitudes específicas en materia social, de prestación de servicios de proximidad y otras análogas, en todo caso se exigirá como requisito de solvencia técnica o profesional la concreta experiencia, conocimientos y medios en las referidas materias.

Volvemos a no poder dejar de señalar la tibieza en la regulación. A nuestro juicio este era uno de los puntos en que cabría añadir una referencia explícita a la necesidad de acreditar el cumplimiento de medidas de igualdad.

En el mismo sentido el art. 127 LCSP regula las etiquetas de tipo social o medioambiental -entre otras las relacionadas con la agricultura o la ganadería ecológicas, el comercio justo, la igualdad de género o las que garantizan el cumplimiento de las Convenciones fundamentales de la Organización Internacional del Trabajo-abriendo la posibilidad de que el órgano de contratación pueda exigirlas pero, nuevamente, restringiéndola a criterios vinculados al objeto del contrato.

Resulta igualmente novedoso el contenido del art. 157.5 LCSP, que prevé la posibilidad de que la mesa de contratación solicite informes que permitan la verificación de las consideraciones sociales y ambientales. En concreto, dispone el precepto se podrán requerir informes a las organizaciones sociales de usuarios destinatarios de la prestación, a las organizaciones representativas del ámbito de actividad al que corresponda el objeto el contrato, a las organizaciones sindicales, a las organizaciones que defiendan la igualdad de género y otras organizaciones para la verificación de las consideraciones sociales y ambientales.

\subsection{El objeto del contrato}

El objeto del contrato se recogía en el TRCSP en el artículo $86^{28}$. El art. 99 LCSP, que viene a substituirlo, añade a lo que este estipulaba:

"el objeto del contrato se podrá definir en atención a las necesidades o funcionalidades concretas que se pretenden satisfacer sin cerrar su objeto a una solución única, en especial, se definirán de este modo en aquellos contratos en los que se estime que pueden incorporarse innovaciones tecnológicas, sociales o ambientales que mejoren la eficiencia y sostenibilidad de los bienes, obras o servicios que se contraten"

Tal vez sea esta la que, a nuestro juicio, constituye la propuesta más innovadora, y que mayores posibilidades ofrece al órgano de contratación abriendo la posibilidad de definir como objeto del contrato la promoción de la igualdad.

No debemos olvidar que, como señala P. VALCÁRCEL FERNÁNDEZ ${ }^{29}$ la definición del objeto del contrato tendrá una incidencia decisiva en todo

${ }^{28}$ G. ZÚÑIGA RIAL. "El tratamiento de las cláusulas sociales en la normativa reguladora de la contratación pública. Su consideración como criterios de adjudicación de los contratos administrativos", Dereito, Vol. 17, n 2, 2008, p.79 
el procedimiento contractual toda vez que la admisibilidad de determinados criterios de adjudicación o de ciertos requisitos de solvencia técnica o profesional dependerá de la vinculación de los mismos al objeto del contrato.

\subsection{Criterios de valoración de las ofertas}

Estamos ante una diferencia fundamental introducida en el nuevo texto legal; la mejor relación calidad precio frente a la valoración de la oferta económica más ventajosa. El artículo 150.1 TRLCSP dice:

"Para la valoración de las proposiciones y la determinación de la oferta económicamente más ventajosa deberá atenderse a criterios directamente vinculados al objeto del contrato, tales como la calidad, el precio, la fórmula utilizable para revisar las retribuciones ligadas a la utilización de la obra o a la prestación del servicio, el plazo de ejecución o entrega de la prestación, el coste de utilización, las características medioambientales o vinculadas con la satisfacción de exigencias sociales que respondan a necesidades, definidas en las especificaciones del contrato, propias de las categorías de población especialmente desfavorecidas a las que pertenezcan los usuarios o beneficiarios de las prestaciones a contratar, la rentabilidad, el valor técnico, las características estéticas o funcionales, la disponibilidad y coste de los repuestos, el mantenimiento, la asistencia técnica, el servicio postventa u otros semejantes. Cuando sólo se utilice un criterio de adjudicación, éste ha de ser, necesariamente, el del precio más bajo."

Frente a esto, el art. 145.2 LCSP señala que "la adjudicación de los contratos se realizará utilizando una pluralidad de criterios de adjudicación en base a la mejor relación calidad-precio" pasando a continuación a enumerar los criterios para evaluarla.

"2. La mejor relación calidad-precio se evaluará con arreglo a criterios económicos y cualitativos. Los criterios cualitativos que establezca el órgano de contratación para evaluar la mejor relación calidad-precio podrán incluir aspectos medioambientales o sociales, vinculados al objeto del contrato en la forma establecida en el apartado 6 de este artículo, que podrán ser, entre otros, los siguientes:

$1^{0}$ La calidad, incluido el valor técnico, las características estéticas y funcionales, la accesibilidad, el diseño universal o diseño para todas las personas usuarias, las características sociales, medioambientales e innovadoras, y la comercialización y sus condiciones;

Las características medioambientales podrán referirse, entre otras, a la reducción del nivel de emisión de gases de efecto invernadero; al empleo de medidas de ahorro y eficiencia energética y a la utilización de energía procedentes de fuentes renovables durante la ejecución del contrato; y al

29 P. VALCÁRCEL FERNÁNDEZ. "Promoción de la igualdad de género a través de la contratación pública". En J.J. PERNAS GARCÍA (Director) Contratación Pública estratégica. Thomson Reuters Aranzadi, Cizur Menor, Navarra, 2013, p.333 
mantenimiento o mejora de los recursos naturales que puedan verse afectados por la ejecución del contrato.

Las características sociales del contrato se referirán, entre otras, a las siguientes finalidades: al fomento de la integración social de personas con discapacidad, personas desfavorecidas o miembros de grupos vulnerables entre las personas asignadas a la ejecución del contrato y, en general, la inserción socio-laboral de personas con discapacidad o en situación o riesgo de exclusión social; la subcontratación con Centros Especiales de Empleo o Empresas de Inserción; los planes de igualdad de género que se apliquen en la ejecución del contrato $y_{\text {, }}$ en general, la igualdad entre mujeres y hombres; el fomento de la contratación femenina; la conciliación de la vida laboral, personal y familiar; ${ }^{30}$ la mejora de las condiciones laborales y salariales; la estabilidad en el empleo; la contratación de un mayor número de personas para la ejecución del contrato; la formación y la protección de la salud y la seguridad en el trabajo; la aplicación de criterios éticos y de responsabilidad social a la prestación contractual; o los criterios referidos al suministro o a la utilización de productos basados en un comercio equitativo durante la ejecución del contrato.

$2^{\circ}$ La organización, cualificación y experiencia del personal adscrito al contrato que vaya a ejecutar el mismo, siempre y cuando la calidad de dicho personal pueda afectar de manera significativa a su mejor ejecución.

30 El servicio posventa y la asistencia técnica y condiciones de entrega tales como la fecha en que esta última debe producirse, el proceso de entrega, el plazo de entrega o ejecución y los compromisos relativos a recambios y seguridad del suministro.

Los criterios cualitativos deberán ir acompañados de un criterio relacionado con los costes el cual, a elección del órgano de contratación, podrá ser el precio o un planteamiento basado en la rentabilidad, como el coste del ciclo de vida calculado de conformidad con lo dispuesto en el artículo 148."

Este nuevo concepto, "mejor relación calidad-precio" ha de entenderse en un sentido amplio. Debe interpretarse la mayor ventaja económica de una proposición no sólo en sentido directo, sino admitiendo las ventajas económicas indirectas con el objeto del contrato, pero directas con los fines públicos perseguidos por el Sector público ${ }^{31}$. En consecuencia, todo criterio de carácter social que haga referencia al proceso específico de producción, prestación, comercialización o de otra etapa de su ciclo de vida cumplirá dicho requisito ${ }^{32}$.

Podríamos así suponer que aún existiendo ofertas que en el sentido estricto de mejor precio fuesen más ventajosas, habría que valorar aquellas otras que cumpliesen objetivos sociales. En este sentido una

\footnotetext{
${ }^{30}$ Sin destacar en el original

31 M. Á. BERNAL BLAY. "Hacia una contratación... op. Cit.

32 E. LARRAZABAL ASTIGARRAGA. "Las cláusulas sociales en la contratación pública y la posibilidad de establecer un salario mínimo en la ejecución de los contratos". Lan Harremanak: Revista De Relaciones Laborales,31, 2014, pp.135-156.
} 
empresa que aportase en su oferta evidencias de cumplimiento de un plan de igualdad estaría presentando una oferta con una mejor calidad precio.

No obstante, hemos de destacar la coincidencia de la nueva Ley con la anterior en un aspecto que no resulta precisamente intranscendente, la referencia al objeto del contrato ${ }^{33}$. Esta tiene vital importancia ${ }^{34}$.

El art 150.1 del TRLCSP ya establecía que, a la hora de valorar la oferta económicamente más ventajosa habría que atender a criterios vinculados al objeto del contrato. La LCSP mantiene esta necesidad de vinculación, y en el art. 145.6 explicita de manera detallada que ha de entenderse por vinculación con el objeto del contrato:

"6. Se considerará que un criterio de adjudicación está vinculado al objeto del contrato cuando se refiera o integre las prestaciones que deban realizarse en virtud de dicho contrato, en cualquiera de sus aspectos y en cualquier etapa de su ciclo de vida, incluidos los factores que intervienen en los siguientes procesos:

a) en el proceso específico de producción, prestación o comercialización de, en su caso, las obras, los suministros o los servicios, con especial referencia a formas de producción, prestación o comercialización medioambiental y socialmente sostenibles y justas;

b) o en el proceso específico de otra etapa de su ciclo de vida, incluso cuando dichos factores no formen parte de su sustancia material."

Aún teniendo en cuenta que las Administraciones Púbicas tienen la obligación de fomentar la competencia en los procedimientos de contratación evitando que se introduzcan en los mismos restricciones injustificadas que la limiten ${ }^{35}$, entendemos que ponderando la posibilidad de colisión de dos derechos, debería haber pesado más el recogido en nuestra Constitución como un derecho fundamental.

\subsection{Las cláusulas sociales como criterios de adjudicación}

EL TRLCSP establecía, en la Disposición Adicional Cuarta preferencias para las proposiciones presentadas por: empresas que tengan en su plantilla un número de trabajadores con discapacidad superior al $2 \%$, empresas de inserción, entidades sin ánimo de lucro y entidades reconocidas como Organizaciones de Comercio Justo; siempre que dichas proposiciones igualen en sus términos a las más ventajosas desde el punto de vista de los criterios que sirvan de base para la adjudicación.

\footnotetext{
${ }^{33}$ Véase la Resolución n660-2018 que en el Fundamento de Derecho cuarto, señala que "Conforme al artículo 145.5.a) de la LCSP los criterios de adjudicación deben estar vinculados al objeto del contrato, no considerando este Tribunal que un plan de igualdad previo de la empresa en su conjunto, guarde esta vinculación"

${ }^{34}$ S. LESMES ZABALEGUI. "Contratación pública y... op. cit

35 M.J. VAÑÓ VAÑÓ. "Limitaciones a la aplicación de cláusulas sociales en la contratación pública desde la perspectiva del derecho de la competencia", CIRIEC-España, Revista de Economía Pública, Social y Cooperativa, 87, 2016, pp.177-202.
} 
Entre todas ellas no se incluía ninguna relacionada con las políticas de igualdad. ${ }^{36}$

La LCSP en su art. 145.2 citado anteriormente, si se refiere a "/os planes de igualdad de género que se apliquen en la ejecución del contrato $y$, en general, la igualdad entre mujeres y hombres; el fomento de la contratación femenina; la conciliación de la vida laboral, personal y familiar"

\subsection{Las clausulas sociales como criterio de desempate}

La LCSP introduce los criterios de desempate, que no aparecían en su predecesora. Los criterios sociales aparecen entre los criterios de desempate susceptibles de ser incorporados a los Pliegos De Clausulas Administrativas Particulares (PCAP)

"Art. 147.1. e) Proposiciones presentadas por las empresas que, al vencimiento del plazo de presentación de ofertas, incluyan medidas de carácter social y laboral que favorezcan la igualdad de oportunidades entre mujeres y hombres".

$Y$ en el caso de que no se hubiese especificado en los pliegos el art. 147.2.c) contempla como criterio de desempate el "mayor porcentaje de mujeres empleadas en la plantilla de cada una de las empresas".

\subsection{Las condiciones especiales de ejecución}

La posibilidad de utilización de cláusulas sociales en las fases de preparación y adjudicación resultan, como hemos visto, un tanto limitadas. Desde que existe la preocupación por la utilización de la contratación pública para la satisfacción de fines sociales, ha sido la fase de ejecución de los contratos la que se ha considerado más adecuada para la inclusión de cláusulas que atendieran a dicho objetivo ${ }^{37}$.

Los artículos 201 y 202 LCSP constituyen una novedad con respecto al $\operatorname{TRCSP}^{38}$. En cuanto a las condiciones especiales de ejecución de carácter social es importante resaltar la obligatoriedad establecida en el art 202.1 de incluir al menos una de las cláusulas sociales

\footnotetext{
36 Vid en este sentido el Acuerdo 45/2016 del Tribunal Administrativo de Contratos Públicos de Navarra que considera nulo el criterio de adjudicación consistente en valorar la presencia de mujeres en el equipo que va a desarrollar el trabajo. Casi simultánea en el tiempo, la Resolución 210/2016 del Tribunal Administrativo Central de Recursos Contractuales considera que la introducción de criterios de adjudicación de carácter social, referidos a la contratación de personas en riesgo de exclusión del mercado laboral y de personas discapacitadas " no resultan contrarias a Derecho porque no introducen elementos distorsionadores de la igualdad y concurrencia ni desvirtúan el principio general de adjudicación a la oferta económicamente más ventajosa, que rigen esta fase de la contratación administrativa, la de adjudicación."

37 M. Á. BERNAL BLAY "Hacia una contratación...op.cit.

${ }^{38}$ En este, las condiciones especiales de ejecución debían estar vinculadas al objeto del contrato, de forma que la finalidad social o medioambiental a promover o fomentar se consiguiese intrínsecamente con la sola prestación del objeto principal. En este sentido se pronunció el Tribunal Catalán de Contratos del Sector Público en su Resolución 44/2017.
} 
"en todo caso, será obligatorio el establecimiento en el pliego de cláusulas administrativas particulares de al menos una de las condiciones especiales de ejecución de entre las que enumera el apartado siguiente".

En este (art. 202.2) se señala que entre otras podrán introducirse condiciones tendentes a

"eliminar las desigualdades entre el hombre y la mujer en dicho mercado, favoreciendo la aplicación de medidas que fomenten la igualdad entre mujeres y hombres en el trabajo; favorecer la mayor participación de la mujer en el mercado laboral y la conciliación del trabajo y la vida familiar; combatir el paro, en particular el juvenil, el que afecta a las mujeres y el de larga duración"

\subsection{Excepciones}

La Disposición Adicional Cuarta, fija un número mínimo de contratos reservados. Será el Consejo de Ministros, o el órgano competente de las Comunidades Autónomas, el encargado de fijar el porcentaje mínimo de estos contratos. Habría sido una nueva oportunidad para incluir alguna referencia a aquellas empresas que tengan una clara apuesta por la igualdad, pero no fue así.

La única cabida la encontramos en las previsiones respecto a la contratación pública en los servicios sanitarios, sociales y educativos recogidas en las Disposiciones Adicionales 47, 48 y 49

Como señala J. M. GIMENO FELIU lo dispuesto en estas implica que un contrato de prestaciones personales de carácter sanitario o social, podría estar excepcionado de las reglas de concurrencia propias de un contrato típico de servicios o productos, dado el marcado carácter estratégico, desde la perspectiva de correcta prestación, del mismo ${ }^{39}$.

\section{LIMITACIONES DE ESTE ESTUDIO}

No podemos dejar de señalar las limitaciones que este estudio presentan por cuestión de espacio y, evidentemente también de tiempo. Sería absurdo pretender por mi parte un estudio exhaustivo de dos leyes que cuentan una con un preámbulo, 334 artículos, 31 disposiciones adicionales, 8 disposiciones transitorias, 6 disposiciones finales y tres anexos, y la otra con un preámbulo, 347 artículos, 53 disposiciones adicionales, 5 disposiciones transitorias, 16 disposiciones finales, más la correspondiente derogatoria de la Ley anterior además de 6 anexos.

Por otra parte hay flecos de esta Ley que todavía no se han desarrollado. El más relevante el Reglamento de Desarrollo que, aunque por su naturaleza no podrá introducir novedades legislativas, si podría aportar matices significativos.

39 J. M. GIMENO FELIU "La nueva ley de contratos del sector público: Una oportunidad para recuperar el liderazgo institucional desde la contratación pública". Observatorio De Contratación Pública,

http://www.obcp.es/index.php/mod.opiniones/mem.detalle/id.317/relcategoria.121/relm enu.3/chk.f d0213b579042f37c80282804a27090a 23/10/2017 Fecha de recuperación $7 / 11 / 2017$ 
Quiero, además hacer una referencia a los Tribunales Administrativos de recursos contractuales que, aún no constituyendo parte del objeto de este trabajo han tenido y es de suponer que sigan teniendo un papel fundamental en la contratación pública.

Tampoco abordé en este estudio las posibilidades que podría aportar la creación de la Oficina Independiente de Regulación y Supervisión de la Contratación (art. 332), en este caso no solo por los motivos anteriores, sino por lo abierto de la redacción del artículo en que se recoge. En virtud de esto el análisis sería meramente de futuribles.

Desde el convencimiento de que la Contratación Pública es el instrumento más potente con que cuentan las Administraciones para implementar políticas públicas, y por tanto un medio idóneo para conseguir que la Ley de Igualdad se cumpla, hemos de reconocer que esta no es una opinión compartida por todos. La propia Comisión Nacional de los Mercados y de la Competencia, en su informe de 16 de julio de 2015, considera que la contratación pública no es el instrumento correcto para el cumplimiento de los objetivos sociales y ambientales de las Directivas, existiendo otros medios más apropiados y menos distorsionadores de la competencia, como exenciones fiscales y modificaciones regulatorias ${ }^{40}$.

No dudamos de que existan otros instrumentos y que una utilización inadecuada de las cláusulas estratégicas no sólo compromete la realización del objetivo político perseguido, sino también la realización de los principios de igualdad, transparencia y eficiencia. Por ello será preciso valorar cuidadosamente el objetivo a perseguir, de forma que se tienda a lograr el deseable equilibrio entre los distintos fines y principios que el procedimiento de licitación ha de armonizar ${ }^{41}$. Pero en ningún modo dejar al margen de la contratación pública las medidas que favorezcan la igualdad.

\section{CONCLUSIONES}

Una primera impresión, a la hora de manejar el texto de esta Ley, ya nos acerca al que, a mi juicio es el principal problema de la misma. La clásica distinción entre criterios de selección y de adjudicación se mantiene. El peso de las medidas encaminadas a conseguir una contratación pública socialmente responsable se ha dejado únicamente a los últimos. Lo preferible sería que las medidas de igualdad hubiesen estado contenidas en las prohibiciones para contratar.

Aparecen, como una novedad, entre los criterios de valoración de las ofertas; pero, al vincularlas al objeto del contrato, parecen más una medida efectista que efectiva.

Estoy convencida de que, después de todas las vueltas que se le han dado a esta ley, de reformas, correcciones, actualizaciones, imposición de transposición de Directivas Comunitarias...se podría haber dado un paso

\footnotetext{
${ }^{40}$ COMISIÓN NACIONAL DE LOS MERCADOS Y DE LA COMPETENCIA (CNMC), Informe IPN/CNMC/010/15, sobre el anteproyecto de Ley de Contratos del Sector Público, de 16 de julio de 2015, p.13

${ }^{41}$ I. GALLEGO CÓRCOLES. "La integración de cláusulas sociales... op.cit.
} 
más. Hubiese sido deseable que la apuesta por la igualdad real hubiese sido mucho más decidida, más clara. Aún sabiendo que no es el objeto de esta Ley, la oportunidad era evidente.

No obstante no todo está perdido. El análisis de la misma me lleva a concluir que, aunque dejándolo excesivamente a la discrecionalidad de los órganos de contratación, la posibilidad está ahí. Si las Administraciones Públicas quieren hacer una apuesta decidida por la igualdad esta Ley les brinda una oportunidad para hacerlo:

- Definiendo el objeto del contrato de manera que entre las necesidades que pretende satisfacer figure la de lograr la igualdad efectiva de hombres y mujeres. Con ello tendríamos la vinculación con el objeto del contrato que parece obsesionar al legislador.

- Especificando en los pliegos de clausulas administrativas particulares la condición de contar con medidas que fomenten la igualdad entre mujeres y hombres.

\section{BIBLIOGRAFÍA}

L. F. AGUILAR VILLANUEVA. GOBERNANZA: El nuevo proceso de gobernar, Fundación Friedrich Naumanm para la libertad, Ciudad de México, 2010.

M. Á. BERNAL BLAY. "Hacia una contratación pública socialmente responsable: Las oportunidades de la ley 30/2007, de 30 de octubre de contratos del sector público". Revista Aragonesa De Administración Pública, 10, 2008, pp. 211-252.

J. BERNETE GARCÍA. "Cláusulas sociales en la contratación pública: Nuevos instrumentos para el fomento del empleo a nivel local". Cuadernos De Trabajo Social, 26, 2013, pp.85-94.

M. BURZACO SAMPER "Contrataciones públicas socialmente responsables: la necesidad de reconsiderar el potencial de la contratación pública en la consecución de objetivos sociales", CIRIEC España, Revista de Economía Pública, Social y Cooperativa, 86, 2016, pp. 281-310.

M.C. CONTE. "Potencial impacto de las compras públicas para el empoderamiento de las mujeres" en "Promoviendo el Desarrollo Inclusivo: Incrementando la Participación de la Mujer en Contratación Pública" Banco Interamericano de Desarrollo - Washington, D.C. 26-27 de mayo de 2015.

I. GALLEGO CÓRCOLES. "La integración de cláusulas sociales, ambientales y de innovación en la contratación pública". Documentación Administrativa: Nueva Época, 4, 2017, pp. 90-113.

J.M. GIMENO FELIU "La transposición de las directivas de contratación pública en España: Una primera valoración de sus principales novedades". Documentación Administrativa. Nueva Época, 4, 2017, pp.7-30.

J. M. GIMENO FELIU. "La nueva ley de contratos del sector público: Una oportunidad para recuperar el liderazgo institucional desde la contratación pública". Observatorio De Contratación Pública, http://www.obcp.es/index.php/mod.opiniones/mem.detalle/id.317/relc 
ategoria.121/relmenu.3/chk.f d0213b579042f37c80282804a27090a 23/10/2017 Fecha de recuperación 7/11/2017

J. M. GIMENO FELIU. "La reforma comunitaria en materia de contratos públicos y su incidencia en la legislación española. Una visión desde la perspectiva de la integridad", en libro colectivo Las Directivas de Contratación Pública, número monográfico especial Observatorio de los Contratos Públicos 2014, Aranzadi, Cizur Menor, Navarra, 2015, pp. 37-105

E. LARRAZABAL ASTIGARRAGA. "Las cláusulas sociales en la contratación pública y la posibilidad de establecer un salario mínimo en la ejecución de los contratos". Lan Harremanak: Revista De Relaciones Laborales, 31, 2014, pp.135-156.

S. LESMES ZABALEGUI. "Contratación pública y discriminación positiva. Cláusulas sociales para promover la igualdad de oportunidades entre mujeres y hombres en el mercado laboral". Lan harremanak: Revista de relaciones laborales, 13,2015, pp.53-86.

J.M. MIRANDA BOTO. "Contratación pública y cláusulas de empleo y condiciones de trabajo en el derecho de la Unión Europea" Lex Social, vol. 6, 2, 2016, pp. 69-91

J. MIRANZO DÍAZ. "Hacia una administración pública sostenible: novedades en la legislación europea de contratación pública" Actualidad Jurídica Ambiental, 64, 2017, pp.1-40

S. MONTENEGRO LEZA. "El antes y el después de la Ley Orgánica 3/ 2007", REDUR, 14, 2016, pp. 117-136.

F. MORATA TIERRA. "Regiones y Gobernanza Multinivel en la Unión Europea", en F. MORATA TIERRA (Ed.). Gobernanza Multinivel en la Unión Europea, Tirant lo Blanch, Valencia, 2004, pp. 19-50.

J. MORCILLO MORENO. "Las clausulas sociales en la contratación pública como garantía frente al dumping social intracomunitario". Revista Aragonesa De Administración Pública, 47-48, 2016, p.p. 180-209

M.M. RAZQUIN LIZARRAGA. "Las nuevas Directivas sobre contratación pública de 2014: aspectos clave y propuestas para su transformación en España", RAP, 196, 2015, pp. 97-13

S. RODRÍGUEZ ESCANCIANO. Cláusulas sociales y licitación pública: análisis jurídico. Instituto Andaluz de Administración Pública, Sevilla, 2009

M. SOLÓRZANO GARCÍA, J. NAVÍO MARCO y R. CONTRERAS COMECHE. "Incorporación de valor social a la contratación pública en España: Situación y perspectivas". Revista De Estudios De La Administración Local Y Autonómica, 4, 2015, pp. 41-50

J. SUBIRATS HUMET. "Si la respuesta es gobernanza, ¿cuál es la pregunta?: factores de cambio en la política y en las políticas", Ekonorniaz. Revista vasca de economía, 24, 2010, pp. 246-269

M.J. VAÑó VAÑó. "Limitaciones a la aplicación de cláusulas sociales en la contratación pública desde la perspectiva del derecho de la competencia", CIRIEC-España, Revista de Economía Pública, Social y Cooperativa, 87, 2016, pp.177-202. 
J.Ma. VALLÈS I CASADEVALL. Ciencia Política. Una Introducción, Ariel, Barcelona, 2006.

P. VALCÁRCEL FERNÁNDEZ. "Promoción de la igualdad de género a través de la contratación pública". En J.J PERNAS GARCÍA (Director) Contratación Pública estratégica. Thomson Reuters Aranzadi, Cizur Menor, Navarra, 2013, pp. 329-368

E.J. VARELA ÁLVAREZ coord. Dirección pública profesional: lecturas para xestionar a era da gobernanza. Escola Galega de Administración Pública. Santiago de Compostela, 2009

G. ZÚÑIGA RIAL. "El tratamiento de las cláusulas sociales en la normativa reguladora de la contratación pública. Su consideración como criterios de adjudicación de los contratos administrativos", Dereito, Vol. 17, 2, 2008, p.65-90 